\title{
Case - Recurrent incarcerated inguinal bladder hernia with small bowel obstruction
}

Jeff McLellan, BComm ${ }^{1}$; Zuhaib M. Mir, MD²; Michael Di Lena, MD $^{3}$;

Sulaiman Nanji, MD, PhD FRCSC ${ }^{2}$; Darren Beiko, MD, MBA, FRCSC ${ }^{3}$

${ }^{1}$ Northern Ontario School of Medicine, Sudbury, ON, Canada; ${ }^{2}$ Department of Surgery, Division of General Surgery, Queen’s University, Kingston, ON, Canada; ${ }^{3}$ Department of Urology, Queen’s

University, ON, Canada

Cite as: Can Urol Assoc J 2019 February 26; Epub ahead of print. http://dx.doi.org/10.5489/cuaj.5740

Published online February 26, 2019

$* * *$

\section{Introduction}

Inguinal hernias are a common surgical complaint, with a lifetime incidence requiring repair of $27 \%$ in men and $3 \%$ in women. ${ }^{1}$ Recurrence of an inguinal hernia requiring repair depends on a number of factors including repair type, surgeon experience, and patient factors, but has been estimated at $1-4 \% .{ }^{2,3}$ The bladder is involved in $1-4 \%$ of inguinal hernias in the general population, and as high as $10 \%$ of cases in men over age $50 .{ }^{4}$ Risk factors for urinary bladder involvement in an inguinal hernia include male gender, age over 50 , and obesity. ${ }^{5}$ The mainstay of treatment for an inguinal bladder hernia is herniorrhaphy.

Most small bladder hernias are discovered intraoperatively due to the lack of urinary symptoms prior to herniorrhaphy. In fact, less than $7 \%$ are diagnosed preoperatively ${ }^{4}$. Small urinary bladder hernias are often asymptomatic, but clinical manifestations can include urinary frequency, urgency, nocturia, hematuria, and doublephase micturition. ${ }^{6}$ Extensive herniation of the bladder, commonly referred to as a scrotal cystocele, is much less frequent. These are more likely to present with urinary symptoms, and urgent intervention is usually required as bladder infarction and/or obstruction may result. ${ }^{6}$ Inguinal bladder hernias with associated small bowel obstruction are rare and rates have not been characterized in the literature.

We present a case of a recurrent inguinal hernia, with bladder and small bowel incarceration, as well as associated small bowel obstruction. In addition, aside from a case involving complete herniation of the bladder, ${ }^{6}$ we present the first such images of a foley catheter balloon being found within the herniated portion of the bladder. 


\section{Case report}

Our patient was an 80-year old male, who presented to the emergency department with a two-day history of vomiting, diarrhea, expressive aphasia, and confusion. Co-morbidities prior to presentation included Alzheimer's disease, prostate adenocarcinoma (T2a, Gleason 6/10, treated with curative radiotherapy), hypertension, gout, previous atrial tachycardia, previously-resected vestibular schwannoma, and interestingly, a previous left inguinal hernia repaired with Shouldice technique. He was seen urgently by the Neurology service, however, acute cerebrovascular pathology was subsequently ruled out. The focus then shifted to the patient's gastrointestinal symptoms, and initial bloodwork revealed a mild leukocytosis $\left(11.2 \times 10^{9} / \mathrm{L}\right)$, elevated creatinine (165 $\left.\mu \mathrm{mol} / \mathrm{L}\right)$, but an otherwise unremarkable hematologic and biochemical profile. Blood cultures and urine culture were drawn and ultimately negative, ruling out underlying sepsis. Initial CT of the abdomen and pelvis demonstrated a recurrent left-sided inguinal hernia containing a loop of dilated small bowel with resultant proximal small bowel dilatation. The hernia also contained a portion of non-opacified bladder with the foley catheter balloon/tip within the herniated segment (figure 1), causing the bladder to remain distended.

The foley catheter balloon was deflated and repositioned. In addition, manual reduction of the hernia was attempted in the emergency department under procedural sedation. Repeat CT images showed successful reduction of the small bowel from the hernia, but persistent incarceration of the urinary bladder within the left inguinal hernia (figure 2). There was no contrast extravasation seen from the bladder, suggesting that its wall was intact.

Nonetheless, given the persistent incarceration of the bladder, as well as the need to assess viability and ischemia via cystoscopy, the patient was consented and booked for urgent operative repair of the hernia. An open plug and patch repair was performed. Intraoperatively, the hernia was noted to be indirect, with no evidence of residual small bowel herniation. Externally, the bladder tissue did not appear to be necrotic or perforated. An intra-operative cystoscopy was also performed, which demonstrated mucosal edema at the dome of the bladder without any necrosis or perforation. A foley catheter was left insitu and the patient tolerated the procedure well.

Follow-up cystogram on post-operative day 8 showed no perforation or filling defects and the foley catheter was removed. Repeat cystourethroscopy at 8 weeks postoperatively demonstrated mild meatal stenosis and bulbar urethral stricture requiring dilation, with minimal inflammation at the site of bladder incarceration but an otherwise well-healed bladder. On follow-up, the patient remained asymptomatic from a bowel and bladder perspective, and was therefore discharged from care. 


\section{Discussion}

Inguinal hernias are a common surgical complaint, with bladder involvement in approximately 1-4\% of cases. While there are many reported cases of inguinal bladder hernias, we believe this to be the first case where the foley catheter balloon was inflated in the herniated portion of the bladder, thereby helping identify the underlying pathology. There is one prior case where a suprapubic catheter balloon was seen in the hernia, however that was a case involving complete bladder herniation. ${ }^{6}$

Small inguinal bladder hernias tend to be asymptomatic from a urinary perspective and therefore, most often discovered intraoperatively which can lead to a more challenging repair and the possibility of bladder injury during herniorrhaphy. Imaging modalities such as CT and cystograms should be considered pre-operatively to clearly delineate the anatomy if there is suspicion for bladder involvement, as well as to guide surgical repair.

When recognized preoperatively, the mainstay of treatment is surgical repair through an open inguinal approach, reducing the bladder to its original location in the pelvis. Partial cystectomy is rarely indicated, except in the case of bladder wall necrosis, herniated bladder diverticulum, tight hernia neck, or bladder tumour within the herniated segment. ${ }^{7,8}$ As such, we recommend routine intra-operative cystoscopy as an easy strategy to avoid missing such pathologies.

In this case, there was also an associated small bowel obstruction. While the small bowel was able to be reduced without operative intervention, incarceration of the bladder segment was an indication for urgent surgery. A review of the literature returns 18 cases of incarcerated inguinal bladder hernias, only two of which had associated small bowel obstructions.

\section{Conclusion}

To the best of our knowledge, this is the first reported case of an incarcerated inguinal bladder hernia where the foley catheter balloon was inflated in the herniated portion of the bladder. The incarcerated small bowel was reduced pre-operatively, however, persistence of bladder incarceration was the indication for operative repair. Given that the herniated bladder tissue was edematous but not necrotic, partial cystectomy was not required and the patient recovered well after herniorrhaphy. 


\section{References}

1. Primatesta $P$, Goldacre MJ. Inguinal hernia repair: Incidence of elective and emergency surgery, readmission and mortality. Int J Epidemiol 1996;25(4):835-39.

2. Magnusson J, Gustafsson U, Nygren J, et al. Rates of and methods used at reoperation for recurrence after primary inguinal hernia repair with Prolene Hernia System and Lichtenstein. Hernia 2018;22(3):439-44.

3. Ramjist J, Dossa F, Stukel T, et al. Reoperation for inguinal hernia recurrence in Ontario: A population-based study. Hernia 2018.

4. Moufid K, Touiti D, Mohamed L. Inguinal bladder hernia: Four case analyses. Rev Urol 2013;15(1):32-6.

5. Oruç MT, Akbulut Z, Özozan Ö, et al. Urological findings in inguinal hernias: A case report and review of the literature. Hernia 2004;8(1):76-79.

6. Bisharat M, O’Donnell ME, Thompson T, et al. Complications of inguinoscrotal bladder hernias: A case series. Hernia 2009;13(1):81-84.

7. Wagner AA, Arcand P, Bamberger MH. Acute renal failure resulting from huge inguinal bladder hernia. Urology 2004;64(1):156-57.

8. Vindlacheruvu RR, Zayyan K, Burgess NA, et al. Extensive bladder infarction in a strangulated inguinal hernia. Br J Urol 1996;77(6):926-27.

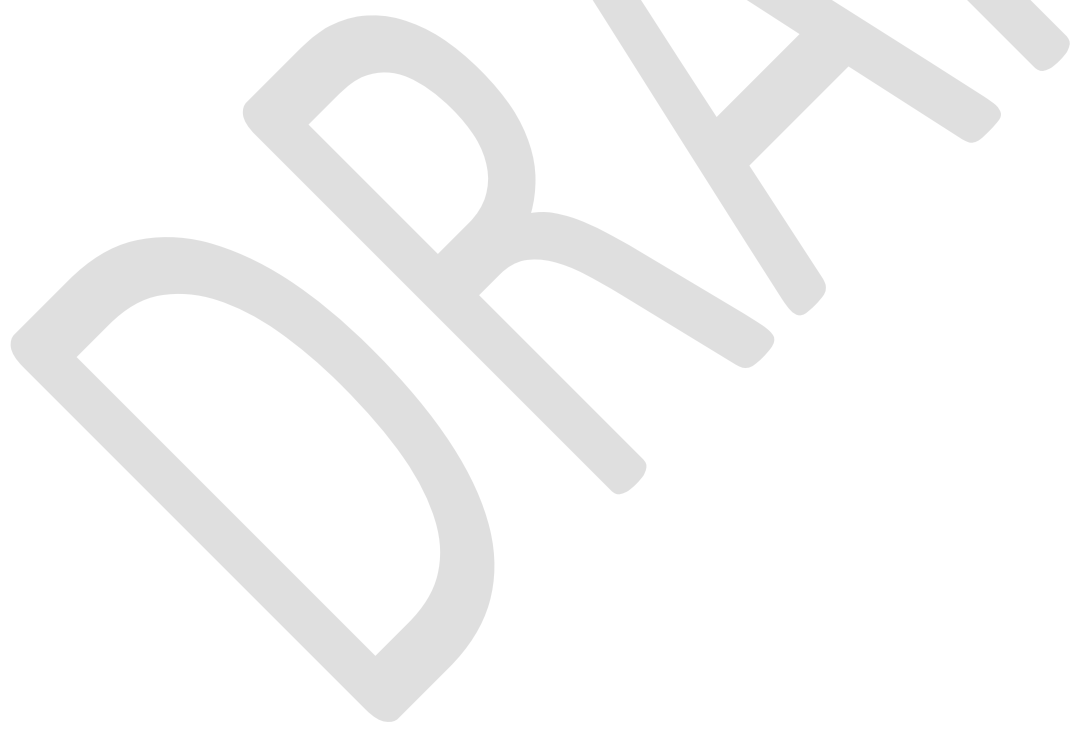




\section{Figures and Tables}

Fig. 1A, 1B. Initial computed tomography scan demonstrating a left inguinal hernia containing a portion of the urinary bladder with the Foley catheter balloon (white arrow) within the herniated segment. Proximal small bowel dilatation is also seen.

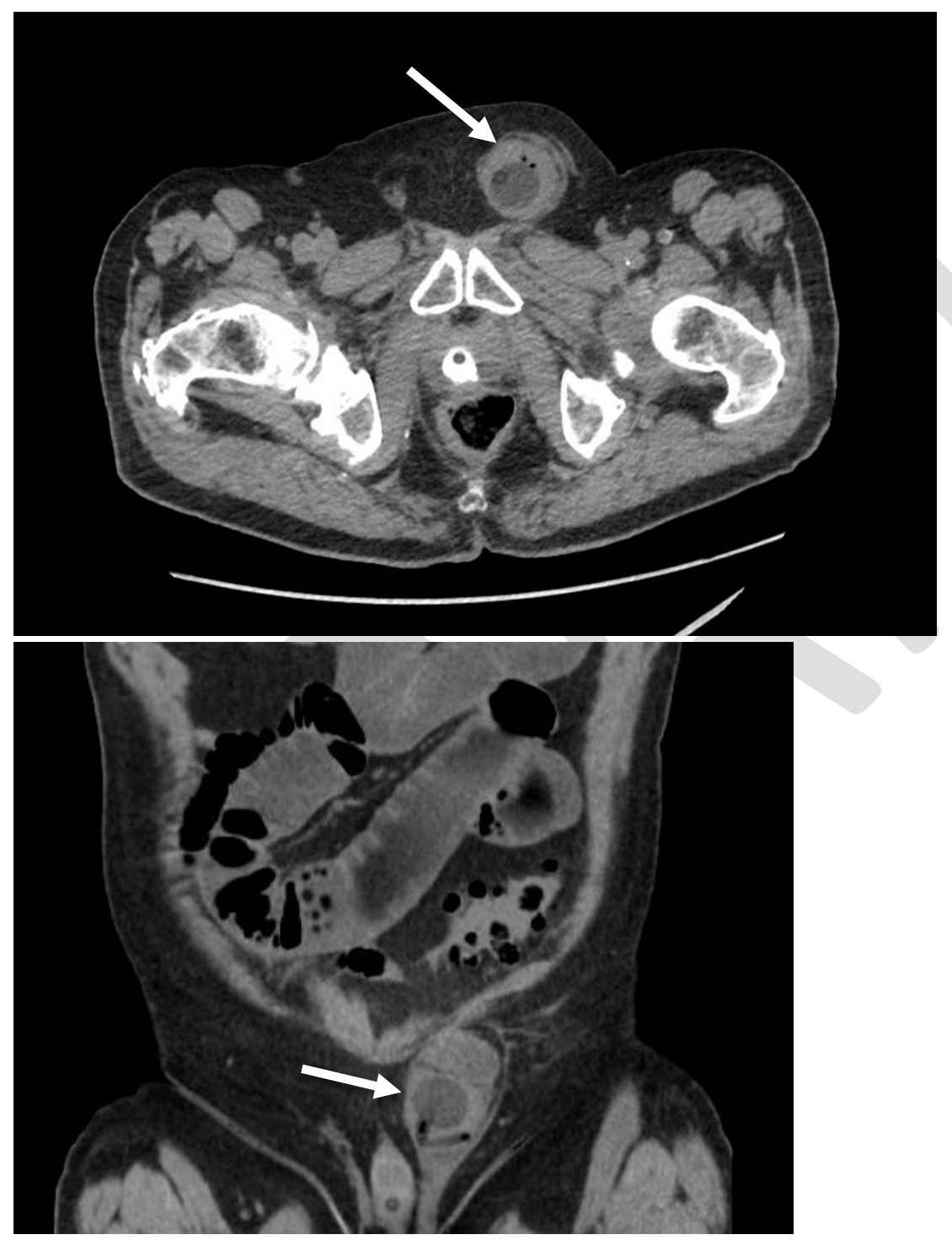


Fig. 2. Repeat computed tomography scan showing persistent incarceration of the urinary bladder and successful reduction of small bowel after bedside reduction attempted under procedural sedation.

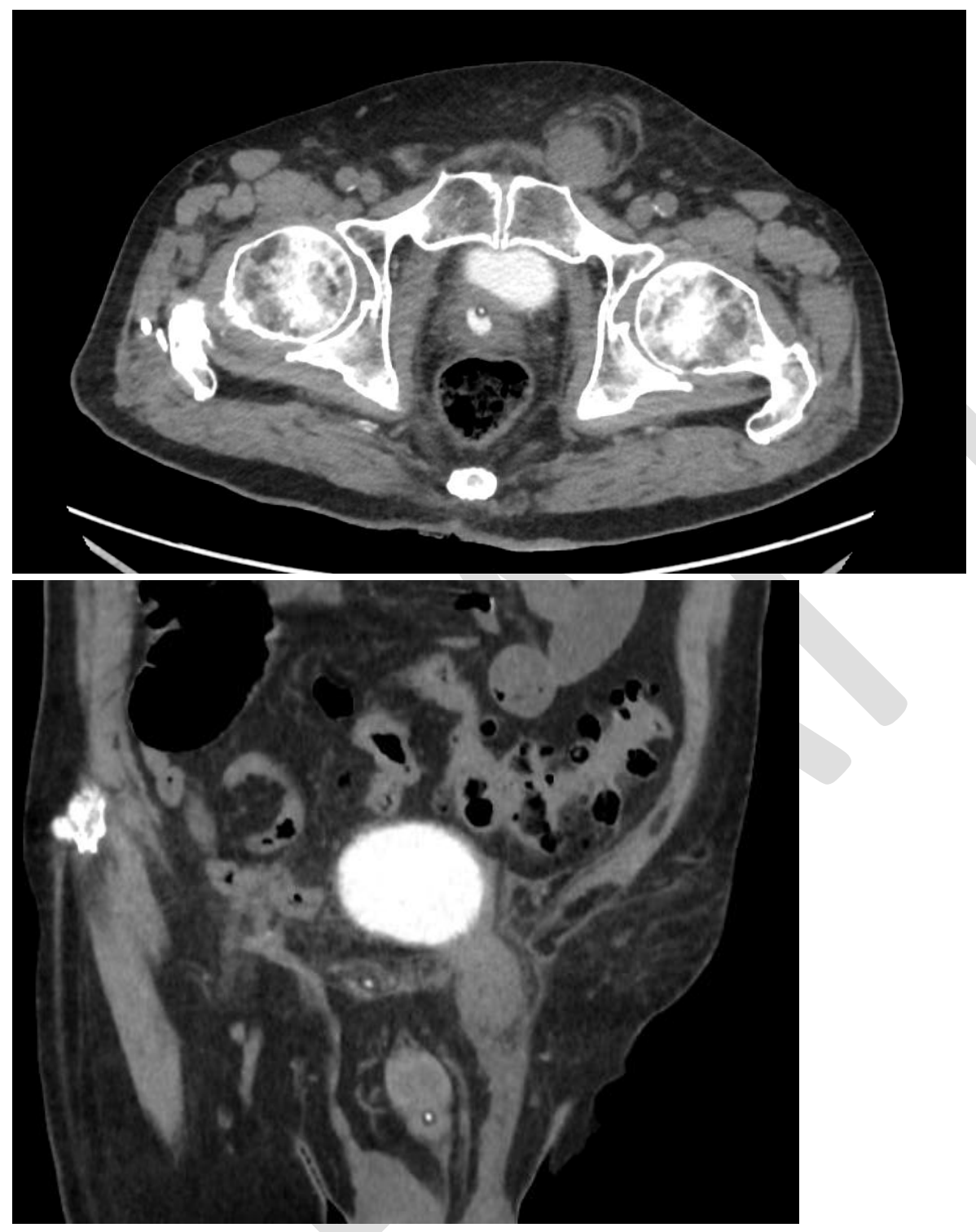

